\title{
CORRECTION
}

\section{Correction to: ACE2, CALM3 and TNNI3K polymorphisms as potential disease modifiers in hypertrophic and dilated cardiomyopathies}

\author{
Amit Kumar ${ }^{1} \cdot$ Bindu Rani $^{1} \cdot$ Rajni Sharma $^{2} \cdot$ Gurjeet Kaur $^{4} \cdot$ Rishikesh Prasad $^{1} \cdot$ Ajay Bahl $^{3} \cdot$ Madhu Khullar $^{1}$ \\ Published online: 28 November 2018 \\ ○) Springer Science+Business Media, LLC, part of Springer Nature 2018
}

Correction to: Mol Cell Biochem (2018) 438:167-174

https://doi.org/10.1007/s11010-017-3123-9

In the original publication of the article, the location and rs number of TNNI3K mouse SNP (3784 C>T) (rs49812611) has been mentioned inadvertently in place of its human homologue. The correct information for human SNP is rs760769780 located at position 74436534 , resulting in $(\mathrm{G}>\mathrm{A})$ change in human TNNI3K gene.

Therefore, TNNI3K (3784 C>T, rs 49812611) should be read as TNNI3K $74436534 \mathrm{G}>\mathrm{A}$, rs number 760769780 in the text. The correct rs number (760769780) and location of gene (74436534) have been provided in the corrected Tables 2 and 3.

The original article can be found online at https://doi.org/10.1007/ s11010-017-3123-9.

Madhu Khullar

madhu.khullar@gmail.com

1 Department of Experimental Medicine and Biotechnology, PGIMER, Lab No 2009, Research Block B,

Chandigarh 160012, India

2 Department of Otolaryngology, PGIMER, Chandigarh, India

3 Department of Cardiology, PGIMER, Chandigarh, India

4 Department of Endocrinology, PGIMER, Chandigarh, India 
Table 2 Genotype frequency of $A C E 2, T N N I 3 K$ and $C A L M 3$ genes in HCM, DCM patients and controls

\begin{tabular}{|c|c|c|c|c|}
\hline Polymorphisms & $\operatorname{HCM}(n=130)$ & Control $(n=236)$ & OR $(95 \% \mathrm{CI})$ & $p$ value \\
\hline \multirow[t]{3}{*}{ ACE2 $(7160726 \mathrm{C}>\mathrm{G})$} & $\mathrm{CC}=81(62.3)$ & $\mathrm{CC}=146(61.9)$ & $0.86(0.53-1.40)$ & 0.62 \\
\hline & $\mathrm{CG}=36(27.7)$ & $\mathrm{CG}=75(31.8)$ & $1.56(0.71-3.44)$ & 0.30 \\
\hline & $\mathrm{GG}=13(10)$ & $\mathrm{GG}=15(6.4)$ & & \\
\hline \multirow[t]{3}{*}{ TNN13K $(74436534 \mathrm{G}>\mathrm{A})$} & $\mathrm{GG}=77(62.3)$ & $\mathrm{GG}=161(68.2)$ & $1.19(0.74-1.89)$ & 0.47 \\
\hline & $\mathrm{GA}=42(32.3)$ & $\mathrm{GA}=74(31.4)$ & $23(2.91-181.38)$ & 0.000056 \\
\hline & $\mathrm{AA}=11(8.5)$ & $\mathrm{AA}=01(0.4)$ & & \\
\hline \multirow[t]{3}{*}{ CALM3 $(-34 \mathrm{~T}>\mathrm{A})$} & $\mathrm{TT}=68(52.3)$ & $\mathrm{TT}=151(63.7)$ & $1.32(0.84-2.08)$ & 0.25 \\
\hline & $\mathrm{TA}=51(39.2)$ & $\mathrm{TA}=86(36.3)$ & Infinite & 0.000048 \\
\hline & $\mathrm{AA}=11(8.5)$ & $\mathrm{AA}=0(0)$ & & \\
\hline Polymorphisms & $\operatorname{DCM}(n=161)$ & Control $(n=236)$ & OR (95\% CI) & $p$ value \\
\hline \multirow[t]{3}{*}{$A C E 2(7160726 \mathrm{C}>\mathrm{G})$} & $\mathrm{CC}=116(72)$ & $\mathrm{CC}=133(56.4)$ & $0.63(0.40-1.00)$ & 0.05 \\
\hline & $\mathrm{CG}=41(25.5)$ & $\mathrm{CG}=74(31.4)$ & $0.15(0.05-0.46)$ & 0.0001 \\
\hline & $\mathrm{GG}=4(2.5)$ & $\mathrm{GG}=29(12.30)$ & & \\
\hline \multirow[t]{3}{*}{ TNN13K $(74436534 \mathrm{G}>\mathrm{A})$} & $\mathrm{GG}=111(68.9)$ & $\mathrm{GG}=161(68.2)$ & $0.8(0.51-1.26)$ & 0.36 \\
\hline & $\mathrm{GA}=41(25.5)$ & $\mathrm{GA}=74(31.4)$ & $13.05(1.63-104.50)$ & 0.002 \\
\hline & $\mathrm{AA}=9(5.6)$ & $\mathrm{AA}=1(0.4)$ & & \\
\hline \multirow[t]{3}{*}{ CALM3 $(-34 \mathrm{~T}>\mathrm{A})$} & $\mathrm{TT}=99(61.5)$ & $\mathrm{TT}=151(63.7)$ & $0.89(0.58-1.38)$ & 0.66 \\
\hline & $\mathrm{TA}=50(31.1)$ & $\mathrm{TA}=86(36.3)$ & Infinite & 0.000023 \\
\hline & $\mathrm{AA}=12(7.5)$ & $\mathrm{AA}=0(0)$ & & \\
\hline
\end{tabular}

Table $3 A C E 2, T N N I 3 K$ and $C A L M 3$ gene allele frequencies in HCM and DCM patients and controls

\begin{tabular}{|c|c|c|c|c|}
\hline Polymorphisms & $\operatorname{HCM}(n=130)$ & Control $(n=236)$ & OR $(95 \% \mathrm{CI})$ & $p$ value \\
\hline \multirow[t]{2}{*}{ ACE2 $(7160726 \mathrm{C}>\mathrm{G})$} & $\mathrm{C}=198(0.76)$ & $C=367(0.78)$ & $1.09(0.76-1.57)$ & 0.64 \\
\hline & $\mathrm{G}=62(0.24)$ & $\mathrm{G}=105(0.22)$ & & \\
\hline \multirow[t]{2}{*}{$\operatorname{TNN} 13 K(74436534 \mathrm{G}>\mathrm{A})$} & $\mathrm{G}=196(0.75)$ & $\mathrm{G}=396(0.84)$ & $1.70(1.17-2.47)$ & 0.005 \\
\hline & $A=64(0.25)$ & $A=76(0.16)$ & & \\
\hline \multirow[t]{2}{*}{$C A L M 3(-34 \mathrm{~T}>\mathrm{A})$} & $\mathrm{T}=187(0.72)$ & $\mathrm{T}=387(0.82)$ & $1.76(1.23-2.52)$ & 0.002 \\
\hline & $\mathrm{A}=73(0.28)$ & $\mathrm{A}=85(0.18)$ & & \\
\hline Polymorphisms & $\operatorname{DCM}(n=161)$ & Control $(n=236)$ & OR $(95 \% \mathrm{CI})$ & $p$ value \\
\hline \multirow[t]{2}{*}{ ACE2 (7160726 C>G) } & $C=273(0.85)$ & $C=340(0.72)$ & $0.46(0.32-0.66)$ & 0.00003 \\
\hline & $\mathrm{G}=49(0.15)$ & $\mathrm{G}=132(0.28)$ & & \\
\hline \multirow[t]{2}{*}{$T N N 13 K(74436534 \mathrm{G}>\mathrm{A})$} & $\mathrm{G}=396(0.84)$ & $\mathrm{G}=263(0.82)$ & $1.17(0.80-1.69)$ & 0.44 \\
\hline & $A=76(0.16)$ & $A=59(0.18)$ & & \\
\hline \multirow[t]{2}{*}{ CALM3 $(-34 \mathrm{~T}>\mathrm{A})$} & $\mathrm{T}=248(0.77)$ & $\mathrm{T}=388(0.82)$ & $1.36(0.95-1.93)$ & 0.08 \\
\hline & $\mathrm{A}=74(0.23)$ & $\mathrm{A}=86(0.18)$ & & \\
\hline
\end{tabular}

